\title{
Darwinian Dynamics of Embodied Chaotic Exploration
}

\author{
Yoonsik Shim \\ Centre for Computational \\ Neuroscience and Robotics \\ University of Sussex \\ Falmer, Brighton, UK \\ y.s.shim@sussex.ac.uk
}

\author{
Joshua E. Auerbach \\ Laboratory of Intelligent \\ Systems \\ École Polytechnique Fédérale \\ de Lausanne \\ Lausanne, Switzerland \\ joshua.auerbach@epfl.ch
}

\author{
Phil Husbands \\ Centre for Computational \\ Neuroscience and Robotics \\ University of Sussex \\ Falmer, Brighton, UK \\ p.husbands@sussex.ac.uk
}

\begin{abstract}
We present Embodied Chaotic Exploration (ECE), a novel direction of research into a possible candidate for Darwinian neural dynamics, where such dynamics are occurring not at the level of synaptic connections, but rather at the slightly higher and more abstract level of embodied motor pattern attractors. Crucially, the (chaotic) neuro dynamics are embodied and it is the whole neuro-body-environment system that must be considered, although the changes occur at the neural level. ECE incrementally explores and learns motor behaviors through an integrated combination of chaotic search and reflex learning. The architecture developed here allows real-time, goal-directed exploration and learning of the possible motor patterns (e.g. for locomotion) of embodied systems of arbitrary morphology. The overall iterative search process formed from this combination is shown to have strong parallels with evolutionary search.
\end{abstract}

\section{CCS Concepts}

-Theory of computation $\rightarrow$ Self-organization; -Computing methodologies $\rightarrow$ Cognitive robotics; Evolutionary robotics;

\section{Keywords}

Evolutionary Computation, Evolution, Cognition, Darwinian Neurodynamics, Neuronal Darwisnism, Robotics

\section{Introduction}

There is a growing body of observations that intrinsic chaotic dynamics exist in the nervous systems of humans and other animals $[5,13,4,20,19]$. Some studies indicate intrinsic chaotic dynamics in animal motor behaviors at both the neural level $[13,19]$ and the level of body and limb movement [14]. These seem particularly prevalent during developmental and learning phases (e.g. when learning to coordinate limbs) [10]. The existence of such dynamics in both normal and pathological brain states, at both global and microscopic

Permission to make digital or hard copies of all or part of this work for personal or classroom use is granted without fee provided that copies are not made or distributed for profit or commercial advantage and that copies bear this notice and the full citation on the first page. Copyrights for components of this work owned by others than the author(s) must be honored. Abstracting with credit is permitted. To copy otherwise, or republish, to post on servers or to redistribute to lists, requires prior specific permission and/or a fee. Request permissions from permissions @ acm.org.

GECCO'16 Companion, July 20 - 24, 2016, Denver, CO, USA

(C) 2016 Copyright held by the owner/author(s). Publication rights licensed to ACM. ISBN 978-1-4503-4323-7/16/07 . \$ \$15.00

DOI: http://dx.doi.org/10.1145/2908961.2931673 scales [20], and in a variety of animals, supports the idea that chaos plays a fundamental role in neural mechanisms [18].

Although the functional roles of chaotic dynamics in the nervous system are far from understood, a number of intriguing proposals have been put forward. Kuniyoshi and Sangawa [7] made the important suggestion that chaotic dynamics underpin crucial periods in animal development when brain-body-environment dynamics are explored in a spontaneous way as part of the process of acquiring motor skills. A few robotics studies have demonstrated that chaotic neural networks can indeed power the self-exploration of brain-body-environment dynamics in an embodied system, discovering self organized patterns that can be incorporated into motor behaviors $[8,6,12]$.

This idea has been successfully applied for generating robot locomotor behaviors $[15,16]$ to allow goal-directed (fitness-directed) search through a general and fully dynamic embodied neural system, whereby chaotic search is exploited through adaptive bifurcations for the real-time, goal-directed exploration and learning of the possible locomotion patterns of an articulated robot of an arbitrary morphology in an unknown environment. This was further extended to explore beyond the fixed set of locomotor behaviors that is initially given by the physical embodiment by allowing the iterative use of embodied chaotic exploration (ECE) through the addition of proprioceptor adaptation [17]. These studies raise an interesting idea about the relationship between fitness-directed ECE and Darwinian neural dynamics [3] and we suggest there is a strong direct analogy between ECE and estimation of distribution algorithms (EDAs) [11, 9], a well-established class of evolutionary algorithms, and that ECE can be viewed as a specialized form of Darwinian neural dynamics.

\section{ECE with Proprioceptor Adaptation}

While many optimization and search strategies use random perturbations of the system variables to search the space of possible solutions, ECE attempts to generate a set of attractors (e.g. locomotor behaviors) that spontaneously emerges from the interactions between the neural system and its physical embodiment. The intrinsic chaotic dynamics of the system are used to naturally power a search process without the need for external sources of noise. The system employs the concept of Chaotic Mode Transition with external feedback [1], which exploits the intrinsic chaoticity of a system orbit as a perturbation force to explore multiple synchronized states of the system, and stabilizes the orbit by decreasing its chaoticity according to a feedback signal that 

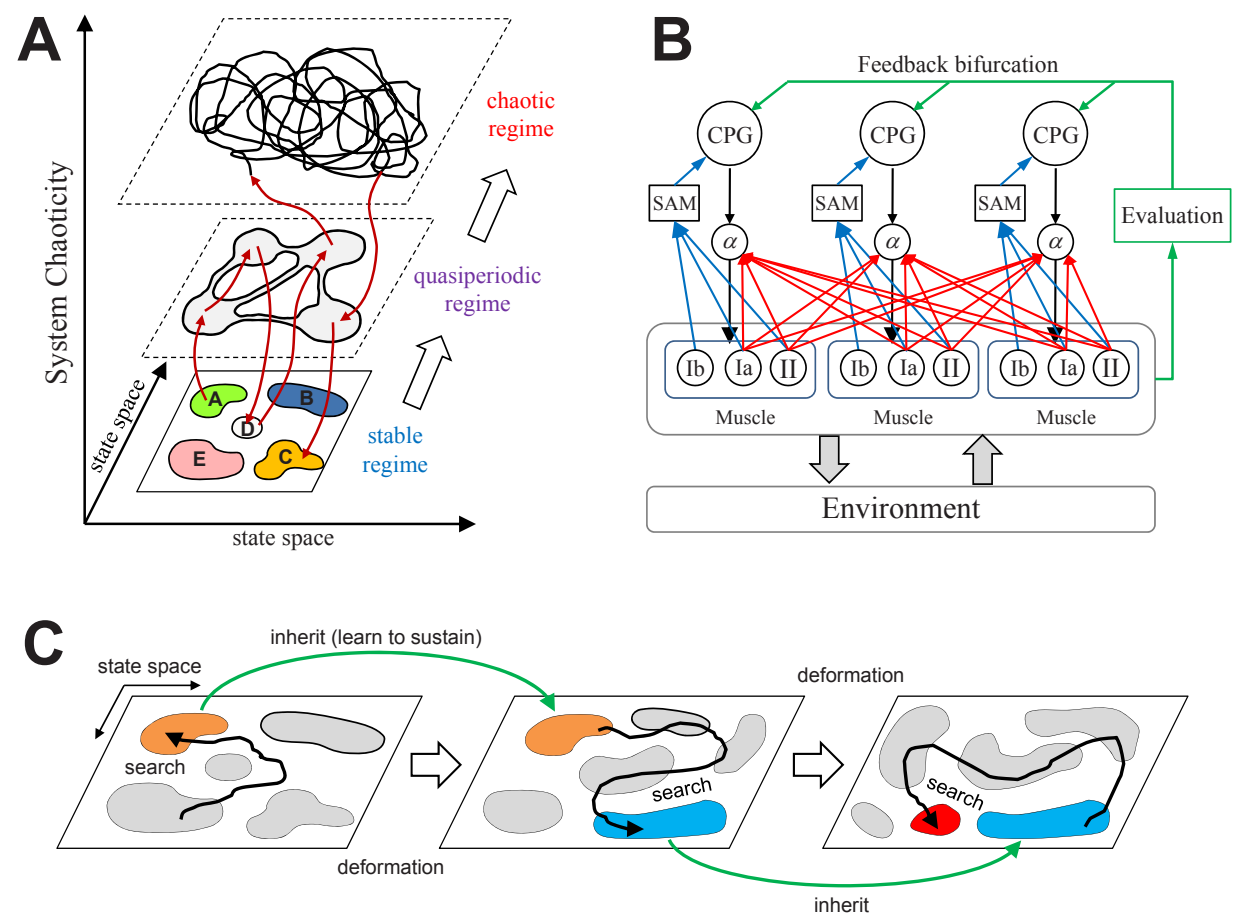

Figure 1: A conceptual illustration of ECE and the schematic diagram of the control system. (A) The state space of a neuro-body-environment system coupled through physical embodiment consists of different basins of attraction with different performance levels. An exploration process finds the desired attractor (attractor C), by varying the complexity of the orbit dynamics through changing the system chaoticity according to the performance feedback. Lump spaces and narrow passages in the landscapes of higher complexities represent quasi-attractors and itinerant pathways, respectively. (B) Schematic diagram of the neural control system for the articulated robot with arbitrary DoFs (3-DoF case is shown). The red connections from muscle proprioceptors (Group Ia, II, and Ib) to alpha motor neurons are learned by Hebbian dynamics in order to sustain (memorize) the phase relationship between the discovered motor pattern and the ongoing sensor signals. (C) Overall dynamics of the repeated exploration-learning through landscape deformation. ECE samples the population of attractors (motor patterns) and explores the patterns by driving the system orbit through the state space. When the orbit is entrained in a high performing basin of attraction, the dynamics is further stabilized by proprioceptor learning. The learning deforms (mutates) the attractor landscape producing a new landscape that inherits major parts of the structure of the previous state space. The process then repeats.

evaluates the behavior (Fig. 1A). Specifically, an evaluation signal, which measures how well the locomotion behavior of the system matches the desired criteria (e.g. locomote as fast as possible), is used to control a bifurcation parameter that alters the chaoticity of the system. During exploration, the bifurcation parameter continuously drives the system between stable and chaotic regimes. If the performance reaches the desired level, the bifurcation parameter decreases to zero and the system stabilizes on this desired behavior.

The overall architecture of the system is illustrated in Fig. 1B. The neural architecture is inspired by the organization of spinobulbar units in the vertebrate spinal cord and Medulla Oblongata (the lower part of the brainstem which deals with autonomic, rhythmic, involuntary functions). The architecture can be applied to a physical system with an arbitrary number of degrees of freedom (one CPG/alpha/muscle group per degree of freedom). Each degree of freedom of the embodied system is controlled by an actuator (muscle) connected to a (alpha) motor neuron, which integrates descending commands from a corresponding central pattern generator (CPG) neuron and proprioceptive signals from all actuators/muscles. Each actuator/muscle conveys three types of proprioceptive signal: group Ia afferents, which measure the rate of change of stretch (or rotation); group II affer- ents, which measure the degree of stretch (or rotation); and one corresponding to the signal from the golgi tendon organ (group Ib), which provides muscle force information. Each CPG unit is fed by each of the three proprioceptive signals from the homonymous muscle/actuator (the blue connections in Fig. 1B). These are integrated and modulated by a sensor adaptation module (SAM) before being passed to the CPG unit. In addition, the group Ia and II proprioceptive signals are fed from all muscles/actuators to each alpha neuron (the red connections in Fig. 1B), which are subject to learning. The CPG units are only coupled indirectly via bodily and environmental interactions (coupling through physical embodiment), which exhibits multiple synchronized states (modes) that reflect the body schema and its interactions with the environment, each of which can be regarded as a potential candidate for meaningful motor behavior. The exploration process, powered by adaptive bifurcation through the feedback evaluation signal, allows the system to become entrained in these modes until one is found that is stable and high performing. The whole process can be interpreted as an iterative, continuous, and deterministic version of stochastic trial-and-error search (for fitter attractors within a given state space), which exploits the intrinsic chaotic behavior of the system. 

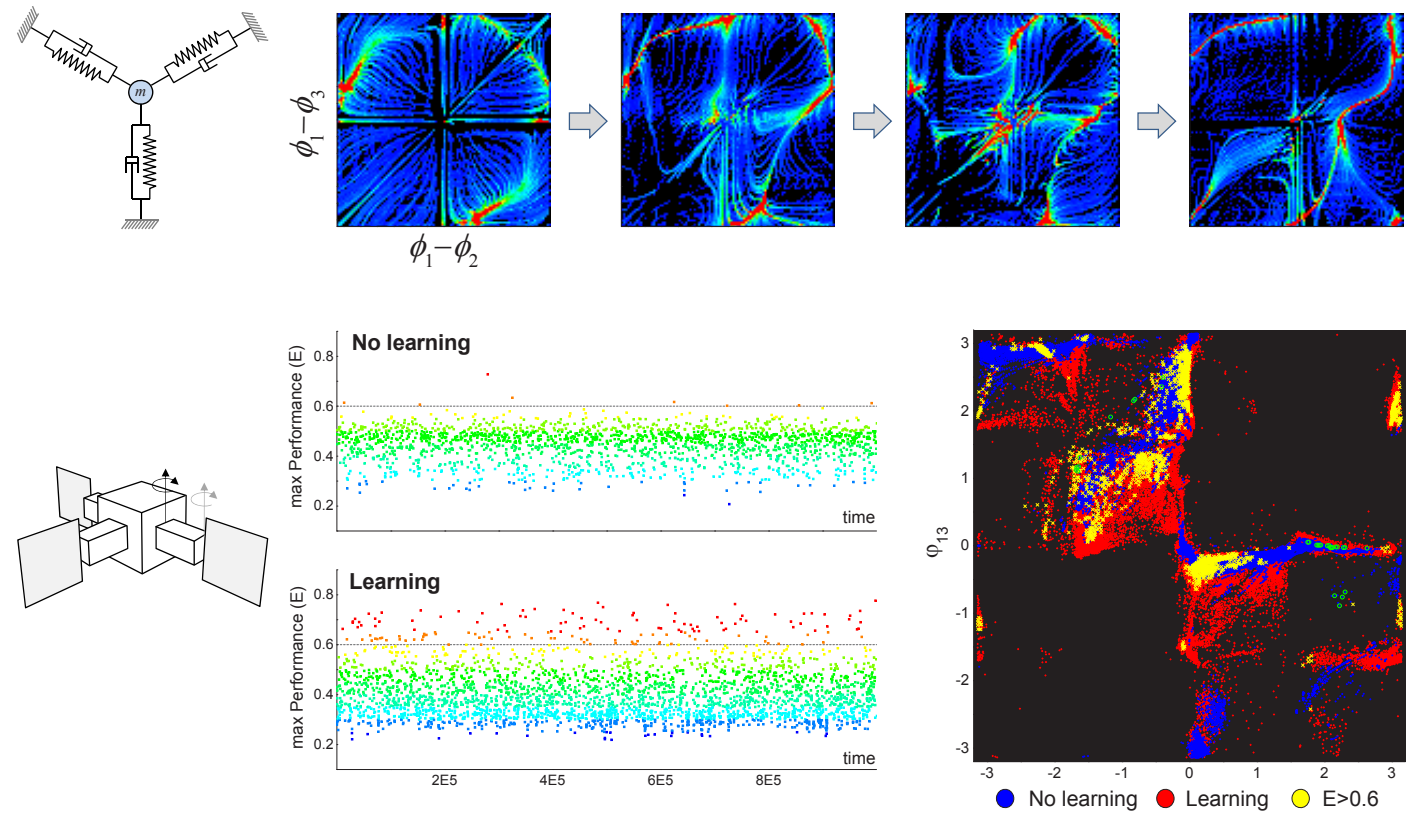

Figure 2: A visualization of the attractor landscape deformation of a simple mass-spring system (top) and the exploration behavior of a three-armed finned swimmer (bottom) [17]. Each spring-damper complex represents the simplified Hill type muscle, and the torsional version of the same muscle model was used for the swimming robot. The $2 \mathrm{D}$ attractor landscapes are drawn by plotting the points from the two phase differences: the difference between the muscles 1 and 2 , and the muscles 1 and 3 for the mass-spring system (colors represent the stability: from blue to red), and the phase differences of the arms 1 and 2, and the arms 1 and 3 for the swimmer (which has three pairs of antagonistic torsional muscles). The performance graphs and the landscape of the swimmer robot depict only the points that the robot can access during the exploration process (see text for further details).

The learning of the afferent weights of the alpha motor neurons are smoothly triggered at the end of each exploration epoch. As the system stabilizes by discovering a useful pattern, the learning of connections between the muscle sensors and the alpha motor neurons are dynamically activated using an adaptive synchronization scheme [2] in order to capture and maintain the discovered motor pattern. This learning process (proprioceptor adaptation) changes the dynamical properties of the embodied system, thus deforming (mutating) its state space (Fig. 1C). The new state space inherits most of the properties of the previous one, but, importantly, new potential pathways for the chaotic exploration process have been opened up, leading to the discovery of new, potentially fitter attractors. The process then repeats until some stopping criteria is met. Thus, exploration and learning are merged as an online continuous dynamical process such that the desired motor behavior is spontaneously explored and discovered in a coherent way through the changing search space by adaptive learning.

Fig. 2 (top) shows examples of the deformation of an attractor landscape by proprioceptor learning using a simple spring-mass system. The attractor landscape of the spring-mass system, which is initially formed by physical embodiment, reveals that a few groups of effective attractors are distributed symmetrically over the phase space, where the three quadrants (1st, 2nd, and 4th) have highly stable group of patterns (near the red points) and one quadrant (3rd) has patterns with the lowest stability (mostly blue). The performances for each quadrant are manually set such that the most unstable region has the highest performance (around the center of 3rd quadrant) to make the overall required behavior suitably challenging. At each explorationlearning epoch, the deformation sequences show that the reflex learning creates/deforms the stable regions (attractor basins) while it generally preserves the regions near the previously selected patterns, until it eventually gets a chance to stabilize the desired regions. The repeated explorationlearning for a freely moving simple underwater robot (Fig. 2 lower) shows that the system visits a wider variety of patterns and finds higher performing patterns than the basic system without the proprioceptor learning. While a sizeable proportion of the newly visited patterns created by reflex learning have a lower performances, a significant portion of them have higher performances than the system without the proprioceptor learning (far denser red dots in higher performances). The discovered location on the landscape plot shows the exploration with learning (red dots) can visit far wider regions that are unable to be accessed by the system without learning.

\section{Relationship between ECE and Evolutionary Neural Dynamics}

The overall ECE process has a number of interesting parallels with evolutionary dynamics. The whole system (literally) embodies a population of (motor behavior) attractors, which are sampled by chaotic exploration. The propriocetor learning process warps (mutates) the state space such that a new landscape of attractors is created, but one that inherits the major properties of the previous (ancestor) landscape (replication with variation). The process repeats with the new population being sampled by chaotic exploration. 
The evaluation mechanism effectively selects a sufficiently fit attractor, which then directly influences the creation of the new landscape through application of the proprioceptor adaptation mechanism.

Since the population of attractors is effectively implicit (the intrinsic dynamics of the system drive it to sample the space of attractors), our embodied system can be thought of as a kind of generative search process. This is loosely analogous to the generative statistical models used by estimation of distribution algorithms (EDAs) [11, 9], which are well established as part of the evolutionary computing canon. Instead of using an explicit population of solutions and the traditional machinery of evolutionary algorithms, EDAs employ a (often Bayesian) probabilistic model of the distribution of solutions, which can be sampled in order to generate possible solutions. Search proceeds through a series of incremental updates of the probabilistic model guided by feedback from the fitness of sampled solutions. In an analogous way, our generative system (the overall system dynamics) is incrementally updated in relation to evaluation based feedback. The overall system dynamics are the generative model, the exploration phase is the sampling step (with the dynamics of the maximum desired performance controlling a selection pressure), and the reflex learning process provides a form of mutation that facilitates the replication (with variation) of the whole phase space, now containing a slightly different population of attractors, but with a bias toward preserving more stable and fitter regions.

The current form of the system is like an ultra elitist evolutionary algorithm: only a single fit member of the population directly influences the next generation, whereas other members only have an indirect effect through influencing the way the population is sampled by chaotic search. An interesting extension, and the focus of further work, will be to incorporate (fitness dependent) influence from a larger sample of the population in creating the new attractor landscape. This will then create solidly Darwinian neurodynamics in a neural architecture that is both practical and fully biologically plausible. This work thus points toward possible fully integrated and intrinsic mechanisms, based entirely on neuro-body-environment interaction dynamics, that might be involved in creating Darwinian processes that could continually run within the nervous systems of future robots [3] without the need for off-line processing or sleight-of-hand magic black boxes.

\section{Acknowledgments}

We acknowledge useful discussions with members of the INSIGHT consortium. Funding: This work was funded as part of the EU ICT FET FP7 project INSIGHT, grant agreement no 308943.

\section{References}

[1] P. Davis. Application of optical chaos to temporal pattem search in a nonlinear optical resonator. Japanese Journal of Applied Physics, 29:L1238-L1240, 1990.

[2] K. Doya and S. Yoshizawa. Adaptive synchronization of neural and physical oscillators. Advances in Neural Information Processing Systems, 4:109-116, 1992.

[3] C. Fernando, E. Szathmáry, and P. Husbands. Selectionist and evolutionary approaches to brain function: A critical appraisal. Frontiers in computational neuroscience, 6(24):1-28, 2012.
[4] W. J. Freeman and G. Viana Di Prisco. EEG spatial pattern differences with discriminated odors manifest chaotic and limit cycle attractors in olfactory bulb of rabbits. In G. Palm and A. Aertsen, editors, Brain Theory, pages 97-119, London, 1986. Springer-Verlag.

[5] M. R. Guevara, L. Glass, M. C. Mackey, and A. Shrier. Chaos in neurobiology. IEEE Transactions on Systems, Man, and Cybernetics, SMC-13:790-798, 1983.

[6] K. Kinjo, C. Nabeshima, S. Sangawa, and Y. Kuniyoshi. A neural model for exploration and learning of embodied movement patterns. Journal of Robotics and Mechatronics, 20(3):358-366, 2008.

[7] Y. Kuniyoshi and S. Sangawa. Early motor development from partially ordered neural-body dynamics: Experiments with a cortico-spinal-musculo-skeletal model. Biological Cybernetics, 95:589-605, 2006.

[8] Y. Kuniyoshi and S. Suzuki. Dynamic emergence and adaptation of behavior through embodiment as coupled chaotic field. In Proceedings of IEEE International Conference on Intelligent Robots and Systems, pages 2042-2049, 2004.

[9] P. Larrañaga and J. Lozano, editors. Estimation of distribution algorithms: A new tool for evolutionary computation. Kluwer Academic Publishers, Boston, 2002.

[10] S. Ohgi, S. Morita, K. Loo, and C. Mizuike. Time series analysis of spontaneous upper-extremity movements of premature infants with brain injuries. Physical Therapy, 88(9):1022-1033, 2008.

[11] M. Pelikan, D. Goldberg, and E. Cantu-Paz. Linkage problem, distribution estimation and bayesian networks. Evolutionary Computation, 8(3):311-340, 2000.

[12] A. Pitti, R. Niiyama, and Y. Kuniyoshi. Creating and modulating rhythms by controlling the physics of the body. Autonomous Robots, 28(3):317-329, 2010.

[13] P. Rapp, I. Zimmerman, A. Albano, G. Deguzman, and N. Greenbaun. Dynamics of spontaneous neural activity in the simian motor cortex: The dimension of chaotic neurons. Physics Letters A, 110(6):335-338, 1985.

[14] M. Riley and M. Turvey. Variability and determinism in motor behaviour. Journal of Motor Behavior, 34(2):99-125, 2002.

[15] Y. S. Shim and P. Husbands. Chaotic search of emergent locomotion patterns for a bodily coupled robotic system. In Proceedings of 12th International Conference on the Synthesis and Simulation of Living Systems (ALIFE XII), pages 757-764, 2010.

[16] Y. S. Shim and P. Husbands. Chaotic exploration and learning of locomotion behaviours. Neural Computation, 24(8):2185-2222, 2012.

[17] Y. S. Shim and P. Husbands. Incremental embodied chaotic exploration of self organised motor behaviours with proprioceptor adaptation. Frontiers in Robotics and $A I, 2(7), 2015$.

[18] C. Skarda and W. Freeman. How brains make chaos in order to make sense of the world. Behavioral and Brain Sciences, 10:161-195, 1987.

[19] D. Terman and J. Rubin. Neuronal dynamics and the basal ganglia. SIAM News,

4(2):http://siam.tekdevelopment.com/oldissues $/ 2007 / \mathrm{march}-2007 /$, 2007.

[20] J. Wright and D. Liley. Dynamics of the brain at global and microscopic scales: Neural networks and the EEG. Behavioral and Brain Sciences, 19:285-320, 1996. 